\title{
Études finno-ougriennes
}

$47 \mid 2015$

Varia

\section{Quelques notes sur l'Oudmourtie en 2015}

\section{Eva Toulouze}

\section{CpenEdition}

Journals

Édition électronique

URL : https://journals.openedition.org/efo/5322

DOI : $10.4000 /$ efo. 5322

ISSN : 2275-1947

Éditeur

INALCO

Édition imprimée

ISBN : 978-2-343-08571-5

ISSN : 0071-2051

\section{Référence électronique}

Eva Toulouze, "Quelques notes sur l'Oudmourtie en 2015 », Études finno-ougriennes [En ligne], 47 | 2015, mis en ligne le 01 juillet 2016, consulté le 20 septembre 2021. URL : http:// journals.openedition.org/efo/5322 ; DOI : https://doi.org/10.4000/efo.5322

Ce document a été généré automatiquement le 20 septembre 2021.

\section{(c) (†) 8}

Études finno-ougriennes est mis à disposition selon les termes de la Licence Creative Commons Attribution - Pas d'Utilisation Commerciale 4.0 International. 


\title{
Quelques notes sur l'Oudmourtie en 2015
}

\author{
Eva Toulouze
}

1 J'ai déjà écrit à plusieurs reprises dans cette revue sur l'Oudmourtie, que je fréquente depuis 1994. Je suis convaincue que c'est bien le travail de terrain sur le long terme qui permet de mettre en évidence bien des éléments importants pour la vie d'une région et de ses habitants.

2 Dans les trois articles que j'ai écrits jusqu'à aujourd'hui sur mes terrains (Toulouze 1995 ; Toulouze 2008 ; Toulouze 2011), je n'ai pas évoqué les moyens d'accès à l'Oudmourtie et je le ferai cette fois-ci.

3 Je tiens à préciser qu'à la différence de mes précédents voyages, j'étais cette fois-ci plutôt inquiète. En effet, ces derniers mois, outre les tensions politiques générales, qui montrent en Russie les Occidentaux comme dangereux, les informations en provenance de Russie étaient de nature à susciter quelques préoccupations: différents cas de chercheurs étrangers expulsés ou sanctionnés pour infraction au régime des visas ont été relevés : il s'agit de chercheurs, voire d'étudiants, qui avaient un visa touristique et qui en profitaient pour travailler en bibliothèque ${ }^{1}$. Les déboires de David Koester, notre collègue anthropologue, accusé d'activités menaçant la sécurité de la Russie, pour avoir organisé une rencontre des derniers Itelmènes parlant la langue, m'inquiétaient. Le fait est que j'avais un visa d'affaires, et que malgré la bonne volonté des institutions qui voulaient m'inviter en Russie, l'établissement d'une invitation a pris tellement de temps que j'ai dû partir en Russie avec le visa qui était sur mon passeport. J’y allais bien pour rencontrer des partenaires, mais on sait bien que s'il y a volonté de créer des problèmes, il sera difficile d'y échapper.

4 Mais il faut dire d'emblée que mes craintes étaient non fondées. Aussi bien aux frontières (à l'aller dans le train Tallinn-Moscou qu'au retour, dans le bus SaintPétersbourg-Tallinn), les autorités frontalières ont été courtoises, voire plaisantes et n'ont posé aucun problème. De même sur place, l'enregistrement (obligatoire dès qu'on passe à un endroit plus d'une semaine) n'a posé aucun problème. De plus, je n'ai pas 
ressenti de tensions particulières auprès de mes interlocuteurs. Mais cela était peutêtre lié aux années de fréquentation...

5 À noter également que les moyens de transport restent confortables. Certes, le train de nuit Tallinn-Moscou, qui j'ai toujours pris pour me rendre en Russie, est menacé : les autorités estoniennes en ont annoncé la fermeture pour la mi-mai. Ce sont des raisons financières qui mettent cette ligne en danger, puisqu'elle est empruntée par un nombre de moins en moins élevé de voyageurs et que la concurrence de l'avion, nettement meilleur marché, se fait sentir. Je me permets d'espérer qu'elle sera promptement rétablie.

6 La ligne Moscou-Iževsk part de la gare de Kazan', sur la place des trois gares (la gare de Leningrad, la gare de Jaroslavl' et la gare de Kazan'), ce qu'il fait qu'il suffit de traverser la place pour gagner la correspondance. Le train que j'ai emprunté sur cette ligne était flambant neuf, avec même la télévision dans les compartiments... Heureusement qu'il était possible de l'éteindre.

7 Pour continuer sur la question des transports, question vitale s'il en est en Russie, à l'intérieur de l'Oudmourtie les dernières semaines d'avril n'étaient pas la période la plus confortable pour se déplacer: en effet on était en pleine période de transition entre hiver et printemps. Cela veut dire que sauf sur les grands axes, asphaltés, le reste des routes de terre étaient une mer de boue, ce qui a fortement limité le déplacement dans les villages. D'ailleurs je me suis rendue compte personnellement cette fois-ci de l'état déplorable des routes de la République d'Oudmourtie, qui contraste fortement avec l'entretien de ces dernières au Tatarstan.

\section{Quelques institutions}

8 Cette fois-ci j'ai consacré l'essentiel de mon temps à la visite d'institutions diverses. Je les passerai en revue, dans l'espoir que mes indications suscitent l'intérêt des lecteurs pour cette région de Russie dont les liens avec les chercheurs français sont devenus traditionnels.

La première institution que j'ai visitée (et où j'ai fait une intervention) est le Musée de Ludorvaj. Ludorvaj est une localité à une dizaine de kilomètres d'Iževsk et qui est connue dans l'histoire de l'Oudmourtie pour une célèbre affaire qui y eut lieu en 1928 et qui a donné le coup d'envoi à la collectivisation des campagnes et à la destruction des anciennes structures de la société paysanne: le conseil paysan, pour une sombre histoire de palissades non entretenues, avait ordonné que quelques paysans fussent fouettés. L'affaire a fait du bruit et le conseil, le keneš, est devenu le symbole de l'archaïsme des anciennes structures traditionnelles ${ }^{2}$. Pour la petite histoire, rappelons qu'en oudmourte, c'était là le terme utilisé pour soviet (qui en russe veut dire conseil) et donc pour soviétique. Il fut vite remplacé par le terme russe... mais aujourd'hui, Ludorvaj n'évoque plus avant tout cette histoire.

C'est là qu'a été ouvert en 1997 un musée en plein air, qui s'est depuis considérablement développé. Sur un territoire traversé par un ruisseau sont éparpillées des fermes de différentes régions d'Oudmourtie : la première et la plus représentative est celle qui nous montre une ferme d'Oudmourtie du Sud avec toutes ses dépendances. Il s'agit d'un terrain appelé "la propriété Atamanov». C'est en effet la maison avec toutes ses dépendances de la famille du linguiste (et ecclésiastique orthodoxe) Mihail 
Atamanov qui y a été transférée. C'est là qu'ont lieu les différentes initiatives, que travaille une partie de l'administration du musée (le reste a un bureau en ville, au centre d'Iževsk), que sont confectionnés les mets oudmourtes avec lesquels les visiteurs sont accueillis.

11 Aujourd'hui le musée comporte d'autres ensembles, dont l'un d'Oudmourtie du Nord (Photo 1) et l'un de paysans russes, sans compter un moulin à vent.

\section{Photo 1}

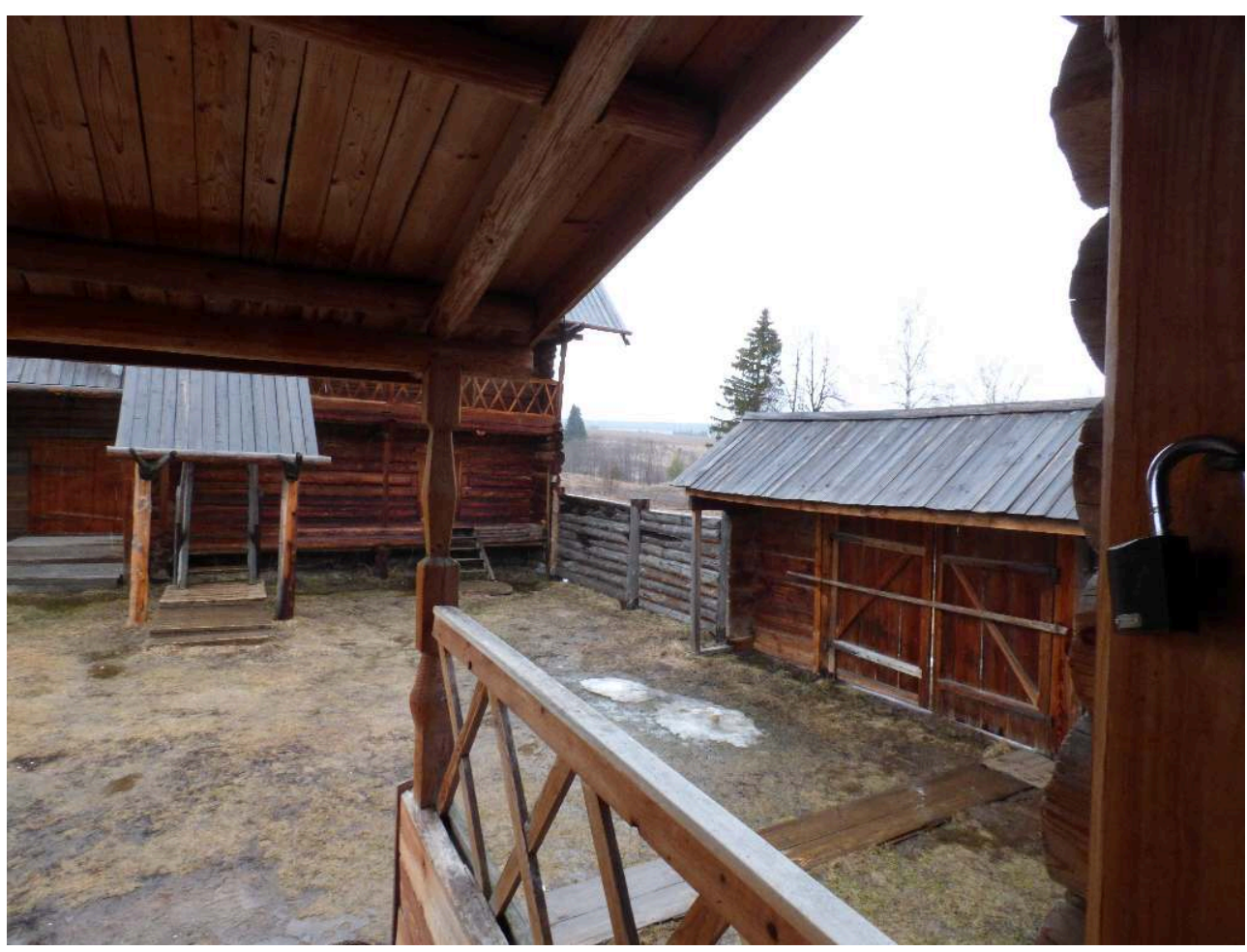

La ferme d'Oudmourtie du Nord au musée en plein air de Ludorvaj.

Photo Eva Toulouze 4/2015

12 Je dois avouer que le temps - la pluie qui tombait sans discontinuer et surtout son corollaire, la boue, qui rendait tout déplacement extrêmement risqué - m'a empêchée de visiter ce musée correctement. Mais il promet, surtout quand la suite des projets aura pu être réalisée, de donner toute satisfaction. On attend en effet encore une ferme bessermane et une ferme tatare. Déjà, cependant, l'accueil d'invités bat son train et d'ores et déjà il est possible d'avoir un aperçu aussi bien de la culture matérielle oudmourte que des chants qu'un groupe d'habitantes âgées de la région se font un plaisir de faire connaître aux visiteurs.

13 La raison de ma présence était l'intérêt que la direction de ce musée porte aux questions religieuses : leur aspiration à avoir un musée ouvert, dynamique, qui montre une culture vivante, ils s'interrogent sur la manière de montrer la religion oudmourte. C'est la raison pour laquelle, au cours d'une "école » saisonnière, ils avaient invité l'archéologue/ethnologue Nadežda Šutina à parler de la typologie des lieux sacrés en Oudmourtie et m'avaient demandé de présenter les cérémonies des Oudmourtes du Bachkortostan. Il faut espérer que cette démarche sérieuse les conduira à présenter les pratiques religieuses oudmourtes de manière respectueuse. 
J'ai été également accueillie à la radio-télévision oudmourte et notamment à la chaine "Moja Udmurtija », qui est la chaîne en langue oudmourte. Elle bénéficie de locaux tout à fait corrects, mais d'un nombre réduit de collaborateurs, dont un journaliste, Anatolij Dobrjakov, qui a été pour moi une révélation : il m'a montré des films qu'il a réalisés avec une grande sensibilité et un grand talent. La direction de la châne est consciente qu'il est un atout pour les Oudmourtes et qu'il faut l'encourager à présenter ses films dans des festivals et à s'y rendre pour élargir son horizon et ses ambitions. Ce n'est sans doute pas un hasard que dans le long entretien que la journaliste Elena Slesarova a réalisé avec moi elle a beaucoup insisté sur l'anthropologie visuelle, qui est un point faible chez les Finno-ougriens de Russie. Or il y aurait beaucoup à faire et il serait particulièrement important et utile que chez tous ces peuples des talents comme celui d'Anatolij Dobrjakov s'épanouissent, pour que nous, spectateurs potentiels, puissions bénéficier de perceptions modernes et sensibles sur leurs cultures. De plus, Moja Udmurtia a des programmes divers en oudmourte (et dans d'autres langues minoritaires, comme en tatare): des informations (15 minutes par jour), des discussions (Maly ke šuono, $15 \mathrm{~min}$ toutes les semaines, avec répétition), des programmes de divertissement (pour adolescents Ogyr bugyr «Secousse»; culinaire, Čečym, deux fois par mois 20 min, programme Sveti doryn kunoyn « En visite chez Sveti », une fois par semaine une heure).

15 Autre institution visitée a été le lycée Kuzebaj Gerd. En réalité, ce lycée était l'hôte d'une conférence régulière des instances de formation des maîtres - «L'enseignement de la langue maternelle dans un espace multilingue». Cette conférence avait acquis un caractère international (ce qui, bien sûr, lui donne une certain prestige supplémentaire) non seulement en raison de ma présence, mais aussi de celle du linguiste autrichien Christian Pischlöger, qui cette année réside en Oudmourtie, et qui tout en maitrisant de manière impressionnante l'oudmourte y a fait une belle présentation plénière sur l'oudmourte sur internet aujourd'hui - qu'il a ouverte avec une citation de Sébastien Cagnoli dans le $\mathrm{n}^{\circ} 44$ de notre revue... Parmi les interventions plénières, je relèverai l'importance de celle d'Irina Vorožcova, qui a insisté sur la nécessité d'établir pour les langues enseignées en Oudmourtie, notamment pour l'oudmourte et le tatare, les niveaux de monitoring correspondant aux standards européens. Cette approche est importante pour rompre l'isolement des langues minoritaires et leur appliquer le même traitement que toutes les autres. Surtout, l'importance de cette conférence était de rassembler des enseignants d'oudmourte et de tatar qui échangent leurs expériences et essayent de promouvoir leurs activités. Ils y sont encouragés par l'active directrice du lycée Anželika Miheeva (Photo 2). 


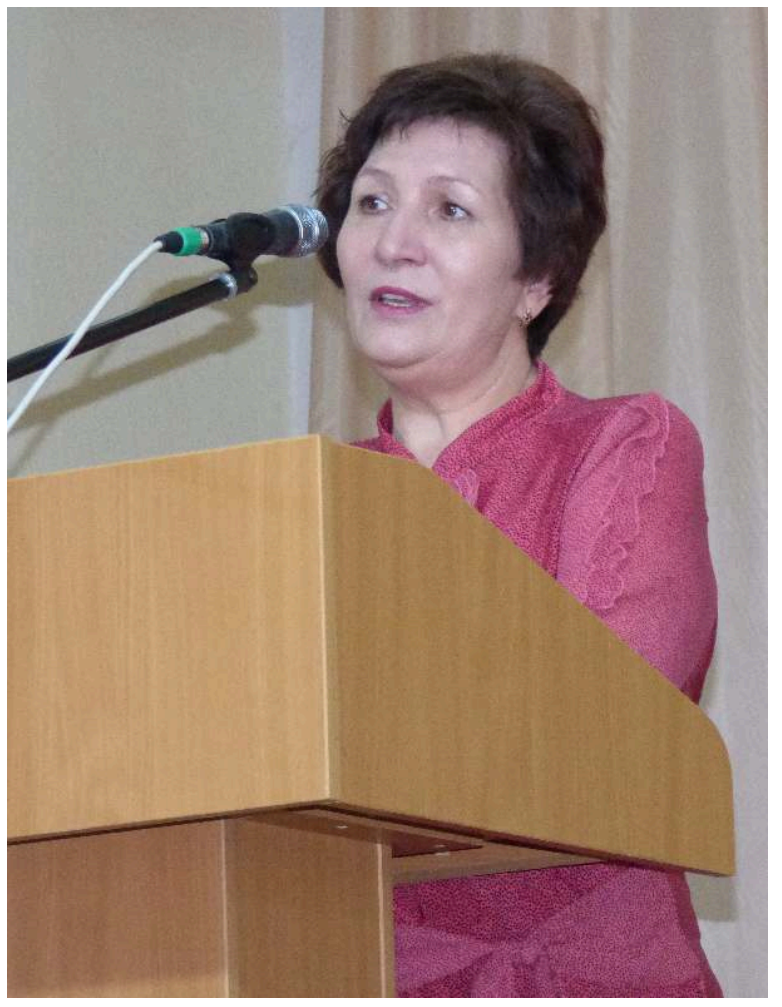

Anželika Miheeva : directrice du lycée Kuzebaj Gerd.

Photo Eva Toulouze, 4/2015.

16 C'est là une personne à suivre : elle se présente aux élections locales pour la ville d'Ǐževsk, où, depuis fort longtemps, il n'y a pas eu d'Oudmourte. Le lycée comprend également un jardin d'enfant pratiquement bilingue, mais en tout cas où l'oudmourte est en bonne place. Elle a voulu faire de son établissement un établissement modèle, où il fait bon étudier et où les nouvelles technologies et les nouvelles méthodes pédagogiques sont à leur place. Les invités ont été impressionnés par le centre des media, animé par les enseignants et par des élèves: ces derniers ont réalisé des entretiens avec les personnalités présentes à la conférence et les ont fait passer sur la radio du lycée après avoir fait le travail d'édition nécessaire. Une hirondelle ne fait pas le printemps, mais elle peut montrer la voie pour s'en rapprocher...

17 J'ai consacré cette fois plus de temps à l'Université d'Iževsk et notamment à sa composante oudmourte, qui est bien sûr celle qui nous intéresse. La doyenne, la linguiste Natalja Kondrat'eva (de même que celle qui l'avait précédée, Ljubov Fëdorova) s'est montrée tout à fait disposée à développer les relations avec nous, et nous avons trouvé une formule qui devrait nous permettre de progresser sur la voie de l'ouverture à l'INALCO de cours d'oudmourte par visioconférence en coopération avec l'Université d'Ǐževsk. On m'a présenté la nouvelle collection d'ouvrages de l'université, UdmurticaUralica, qui a lancé son premier volume, en l'honneur de l'éminent linguiste Valej Kel'makov, en 2014. Par ailleurs, une enseignante d'histoire, Olga Mel'nikova, responsable des masters, a exprimé son désir de nous voir participer au programme de master finno-ougrien. Ceci exprime clairement la volonté des universitaires oudmourtes de se désenclaver et de développer leurs liens internationaux. Comme le prouvent également les cours d'été, qui ont leurs aficionados qui y retournent tous les 
ans. De Paris, il y a déjà eu trois participants (Guillaume Enguehard, Martin Carayol, Charles Thibeault) et j'espère qu'il y en aura d'autres. J'ai cependant rencontré également le département d'études françaises, avec Tamara Zelenina et Anastasia Miftahudtinova qui ont entrepris une coopération avec la faculté de philologie oudmourte mutuellement avantageuse. Leur désir d'accueillir des lecteurs de français peut ouvrir des possibilités pour les étudiants de l'INALCO intéressés par le monde oudmourte.

Enfin, dernière institution à laquelle j'ai consacré de mon temps, l'Institut oudmourte d'histoire, de langue et de littérature, filiale de la section ouralienne de l'Académie des Sciences de Russie. Non seulement j'ai rencontré divers chercheurs avec lesquels nous entretenons des relations proches de coopération, notamment la sociologue Galina Nikitina, spécialiste des campagnes oudmourtes aussi bien dans l'histoire qu'aujourd'hui, mais j'ai eu un long entretien avec le directeur Aleksej Zagrebin. Il m'a tenue informée des réformes actuelles de l'Académie des Sciences, qui s'inscrivent dans le mouvement général qui touche l'ensemble des établissements de recherche du monde, bien au-delà de la Russie: tendances à la concentration, application aux sciences humaines des mêmes critères qui régissent les sciences « dures", chasse à la visibilité et aux publications sur des listes « reconnues » (et de ce point de vue là, la Russie ne reconnaît pas les institutions européennes: ERIH ne compte pas, seul l'américain Web of Science est reconnu sur la liste dite « vak» des revues acceptables). Par exemple, pour l'instant aucun des chercheurs de l'institut dont les communications ont été acceptées pour le Congrès d'Oulu des finno-ougristes, CIFU XII, ne sait s'il/elle pourra se rendre en Finlande. L'institut pour l'instant tient bon, mais son directeur s'attend à des vagues de licenciements et à des périodes encore plus difficiles. Entretemps, quelques beaux projets les maintiennent à flot. Notamment un projet de publication de textes et de recherches "cachés » dans le domaine finno-ougrien: il s'agit de documents d'archives collectés après la première guerre mondiale auprès des prisonniers de guerre et d'autres trésors qui sont depuis restés à l'ombre des archives.

\section{La vie des villages}

19 Comme à l'accoutumée, j'ai tenu à passer du temps dans quelques villages. Tout d'abord, mes séjours sont l'occasion de renforces mes liens avec des personnes que je connais depuis, pour la plupart, pratiquement vingt ans. Comme je l'ai souligné au début de ce texte, le travail sur la longue durée est pour moi une méthode très importante. Au-delà même de la dimension professionnelle, la fidélité en amitié est une valeur à laquelle je tiens. Cette fois-ci, j'avais peu de temps. Mais je n'avais pas été en Oudmourtie depuis quatre ans, laps de temps que je n'avais jamais laissé auparavant s'allonger autant. Il était donc important que je retourne sur ces chemins depuis longtemps balisés, mais que j'avais négligés ces dernières années.

\section{Izgurt (Kamennyj)}

20 J'ai mentionné dans ma chronique de 2011 que je loge toujours chez des amis au village d'Izgurt, à $8 \mathrm{~km}$ de la capitale Iževsk. Je ne vais pas répéter les informations que j'ai déjà publiées dans cette revue. Les personnes sont les mêmes, avec quatre ans de plus. Et le quartier lui aussi a quatre ans de plus. J’avais logé, la dernière fois que j'avais été 
en Oudmourtie, à l'été 2011, dans le kenos, une ancienne grange transportée dans cette belle localité surplombant une vallée. Ayant figé le temps dans mon imagination, j'avais pensé que je serais de nouveau hébergée au même endroit. Mais en quatre ans les choses changent : à côté Juri avait construit sa maison et c'est là que j'ai été accueillie avec tout le confort urbain envisageable, et en plus le sauna et le sentiment d'être dans village (Photo 3).

Photo 3

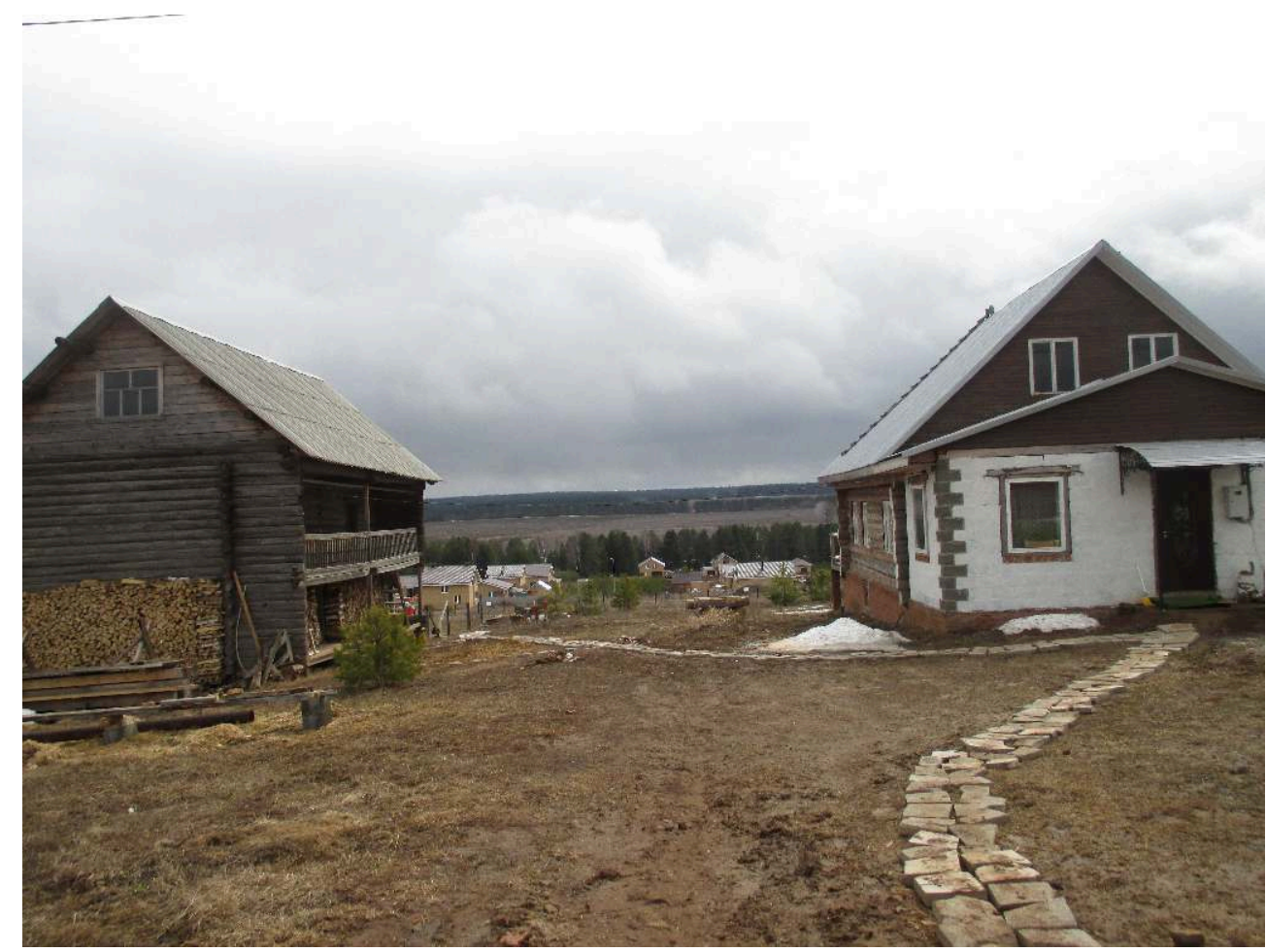

Village d'Izgurt, près de la capitale. À gauche, le kenos traditionnel oudmourte. À droite, la nouvelle maison.

Photo Eva Toulouze, 4/2015.

21 En même temps, ce sentiment est moins authentique qu'il l'était il y a quatre ans : en effet, la vallée s'est remplie et un nouveau quartier de maisons résidentielles y a poussé.

D'ailleurs, en Oudmourtie comme ailleurs en Russie, tout le monde construit (voir dans ce numéro, l'article de Svetlana Russkih). Avoir une maison, telle est l'aspiration de tout le monde ; d'ailleurs, un élément central de l'identité masculine est la construction de la maison et la capacité de faire face à cette tâche. Et une fois que la maison est construite et qu'on y vit, ce n'est pas terminé. Car il faut assurer l'avenir de ses enfants. Peut-être certaines familles particulièrement riches peuvent-elles envisager d'acheter un logement tout prêt pour ses enfants. Mais la plupart des gens cherchent un terrain à construire et consacrent une partie importante de leurs ressources et des énergies qui leur restent à la retraite à aider leurs enfants (Photo 4). 


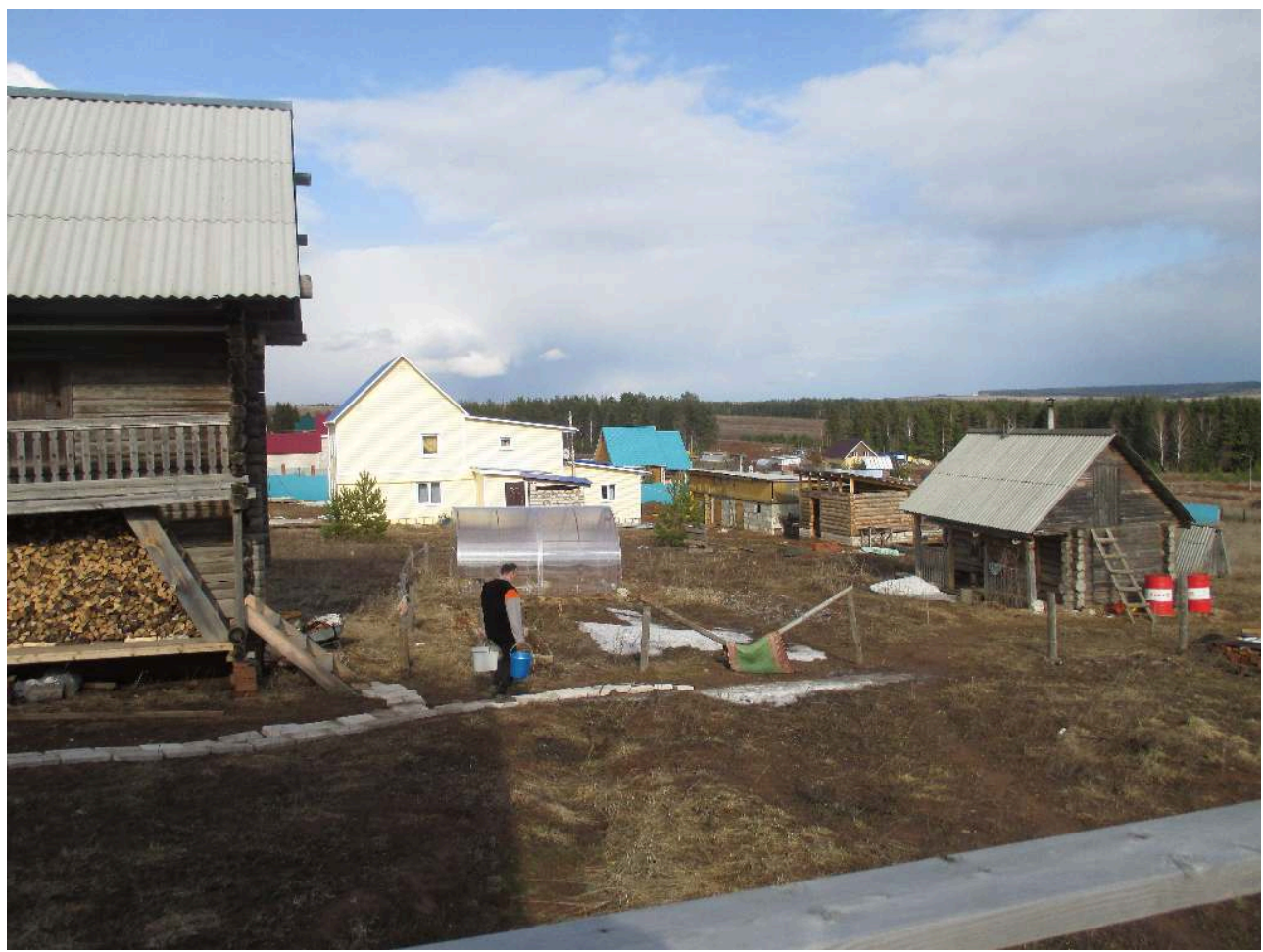

Vue sur les nouveaux quartiers en construction du village d'Izgurt.

Photo Eva Toulouze, 4/2015.

J'avais évoqué dans mon article de 2011 la cérémonie que Sanko avait faite en souvenir de mon ancien compagnon de voyage décédé en 2010. Je disais : «quelques jours plus tard, quand nous rentrerons à Iževsk, Sanko m'annoncera qu'entre-temps il avait terriblement plu et que la pluie avait dévasté le potager et de manière générale les terres sur lesquelles Jurij construisait. Il m'a dit que sa femme, Ljuba, lui avait reproché d'avoir fait la petite cérémonie : il n'avait aucune raison (à son avis) de la faire et il avait déchaîné les foudres des dieux" (Toulouze 2011, p. 255). Nous sommes revenus sur cette péripétie lors de ma rencontre avec son ex-femme Ljuba et il a été intéressant de noter que notre discussion a permis d'y voir plus clair, du moins conformément à la vision oudmourte du monde. En effet, Sanko avait cru que mon ami avait été emporté par l'océan et qu'il n'avait pas été retrouvé. Or une vague l'avait projeté contre des rochers et il s'était noyé en conséquence, mais le corps avait été retrouvé. Sanko avait dit des paroles inadéquates. Les deux, Sanko et son ex-femme ont convenu que c'était là la raison de l'inondation : il s'était montré irresponsable en accomplissant un rituel qui ne correspondait pas à la réalité. Un certain ordre était rétabli dans le monde : tout s'expliquait par une erreur humaine.

\section{Udd'jadi (Karamas Pel'ga)}

Pour moi, la visite de cette fois-ci à Uddjadi (Photo 5) a été en même temps courte, satisfaisante, intéressante et attristante. 


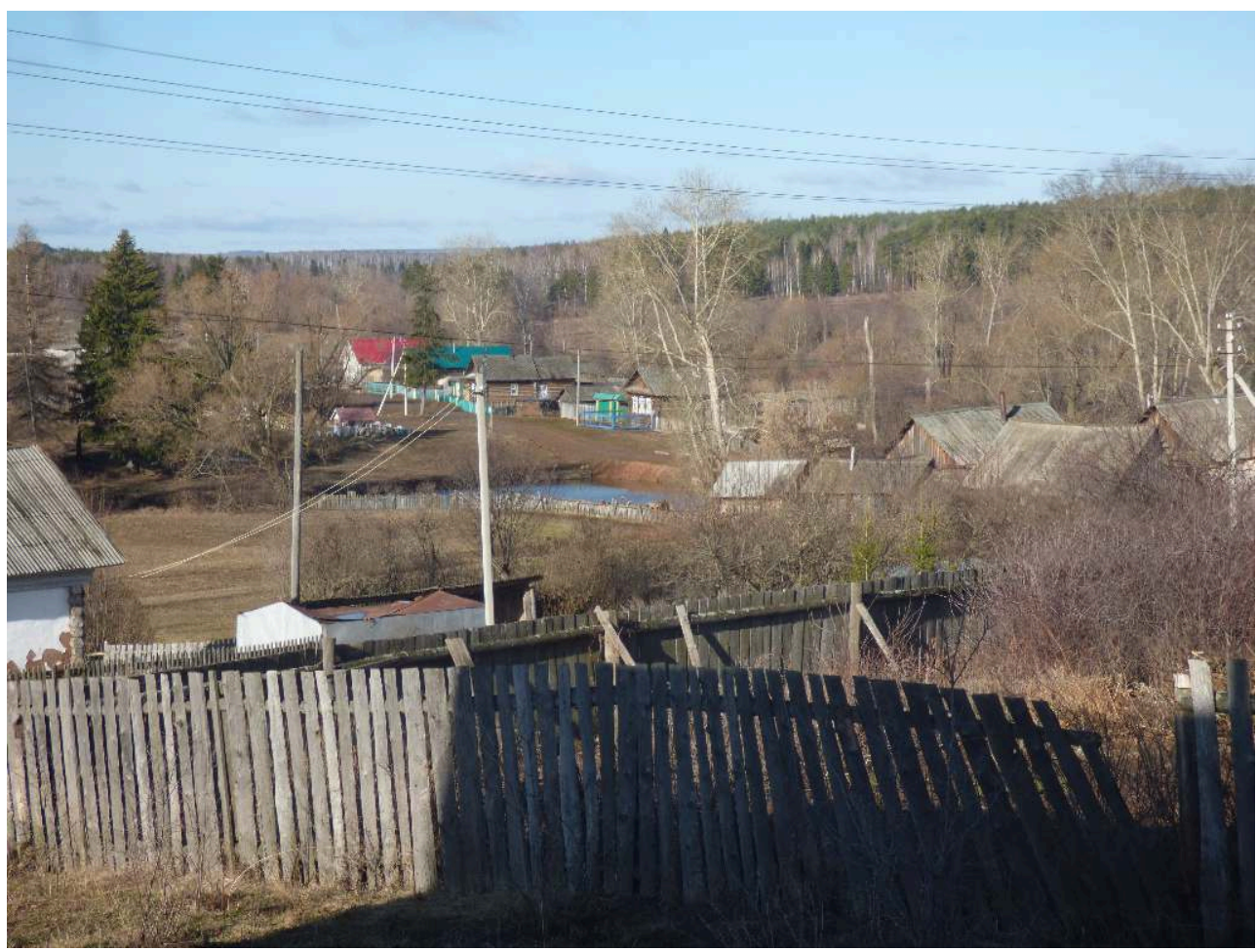

Vue du village d'Uddjadi (Karamas Pel'ga), dans le raïon de Kijasovo.

Photo Eva Toulouze, 4/2015.

Une visite courte : je n'ai pu rester qu'une soirée et une matinée. Elle a été attristante, parce que ma vieille amie olga est aujourd'hui grabataire. Elle est tombée chez elle, s'est cassée une jambe et celle-ci ne s'est pas remise, sans doute en raison du diabète qui l'afflige. C'est bien sûr triste de voir quelqu'un d'aussi énergique et vital qu'olga dans cet état, d'une maigreur à faire peur. Et surtout de sentir qu'elle n'est plus qu'un poids pour les autres, alors qu'encore il y a deux ou trois ans, c'était elle qui faisait marcher la maison.

Mais elle a été aussi satisfaisante, car la vie continue: Irina et Sacha ont eu un deuxième garçon, un petit Aleksej de quatre ans et la maison a été réaménagée pour permettre à une famille de vivre confortablement: ainsi deux chambres ont été ajoutées à la maison, en redistribuant l'espace. Sacha travaille à l'organisme pour l'emploi au chef-lieu du raïon, Kijasovo, où il se rend tous les jours en autostop. Irina est enseignante à l'école du village. Mais sa formation ne convient pas vraiment : elle a été formée à enseigner l'oudmourte et le finnois, mais il y a déjà un enseignant d'oudmourte, plus âgé qu'elle, et elle se retrouve à enseigner le russe. Pour mettre ses diplômes en conformité avec son travail (elle risque d'être licenciée en cas d'inspection), elle a entrepris de faire des études de russe à l'université d'Iževsk, où elle va plusieurs fois par an passer quelques semaines. Les enfants sont joyeux et en bonne santé, ils sont bilingues, même si la famille parle oudmourte à la maison. Mais ils passent beaucoup de temps, malgré leur jeune âge (six et quatre ans) sur l'ordinateur et sur internet. La ferme prospère. Sacha sent clairement le manque d'une compagnie masculine - c'est pourquoi il profite de la venue de tous les visiteurs pour passer bien 
du temps à fumer et à leur faire faire toutes sortes de travaux qu'il n'arrive pas à faire tout seul.

Elle a été intéressante, car un pan nouveau de la vie de ce village s'est ouvert à moi, qui n'était pas visible il y a quatre ans. C'est en effet en 2010 que s'est ouvert ici un Centre de la culture oudmourte (Photo 6).

\section{Photo 6}

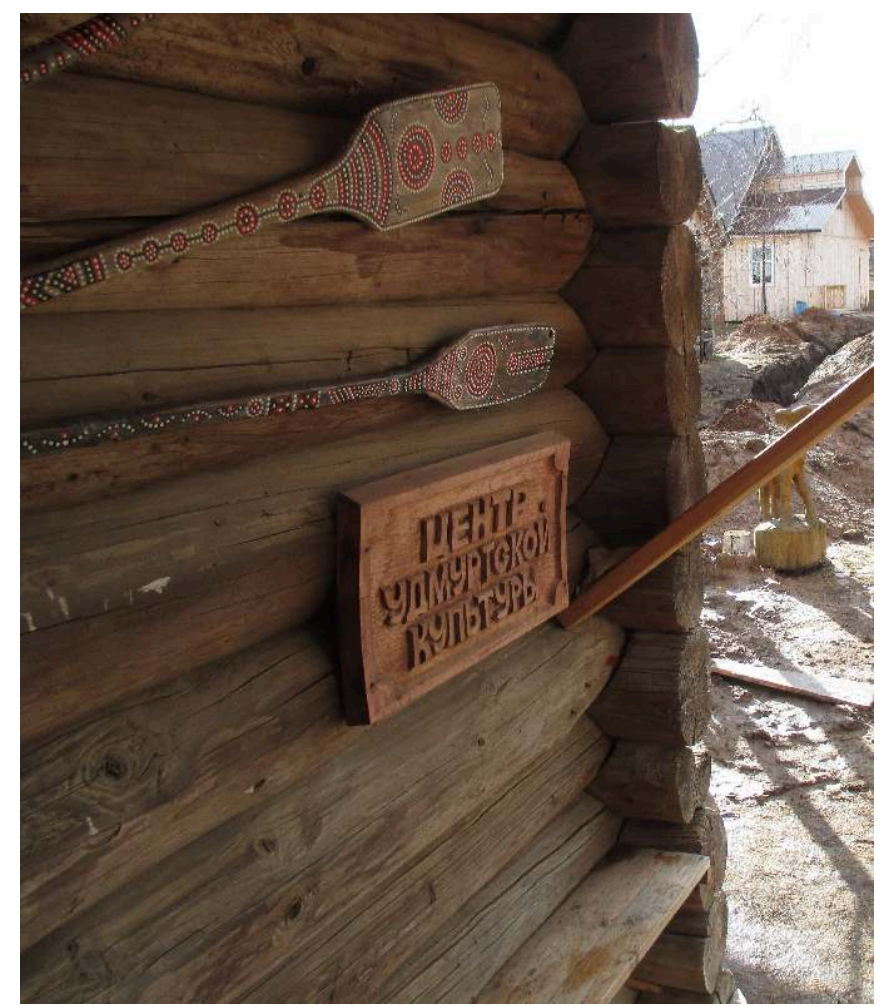

Le Centre de la culture oudmourte ouvert en 2010 à Uddjadi.

Photo Eva Toulouze, 4/2015.

Il a été ouvert dans la maison d'une famille apparentée à celle d'olga (une de ses sœurs s'est mariée dans cette famille). C'était une maison qui avait un sanctuaire domestique qui a fonctionné jusque dans les années 1950. Il était dans la cour de la maison, et c'est le père de famille qui y officiait, le père d'une dame appelée Vassa qui l'anime aujourd'hui avec la nièce d'olga, Elena, que j'avais mentionnée en passant en 2011. Ce sanctuaire, la kuala, a été détruit par un responsable local du parti, qui dans les années 1950 avait décidé de mettre un terme aux «superstitions» des villageois. Il l'avait réduit en bois de chauffage, alors qu'une autre kuala encore en fonctionnement au village, appartenant à un autre clan, avait été transportée au chef-lieu, où elle avait brûlé dès le lendemain. Il semblerait que le responsable du parti soit mort quelques semaines plus tard. Le sanctuaire, lui, a été reconstruit, mais aujourd'hui il ne fonctionne plus en tant que tel: on peut toujours le voir dans la cour de la maison (Photos 7 et 8 ) 
Photo 8

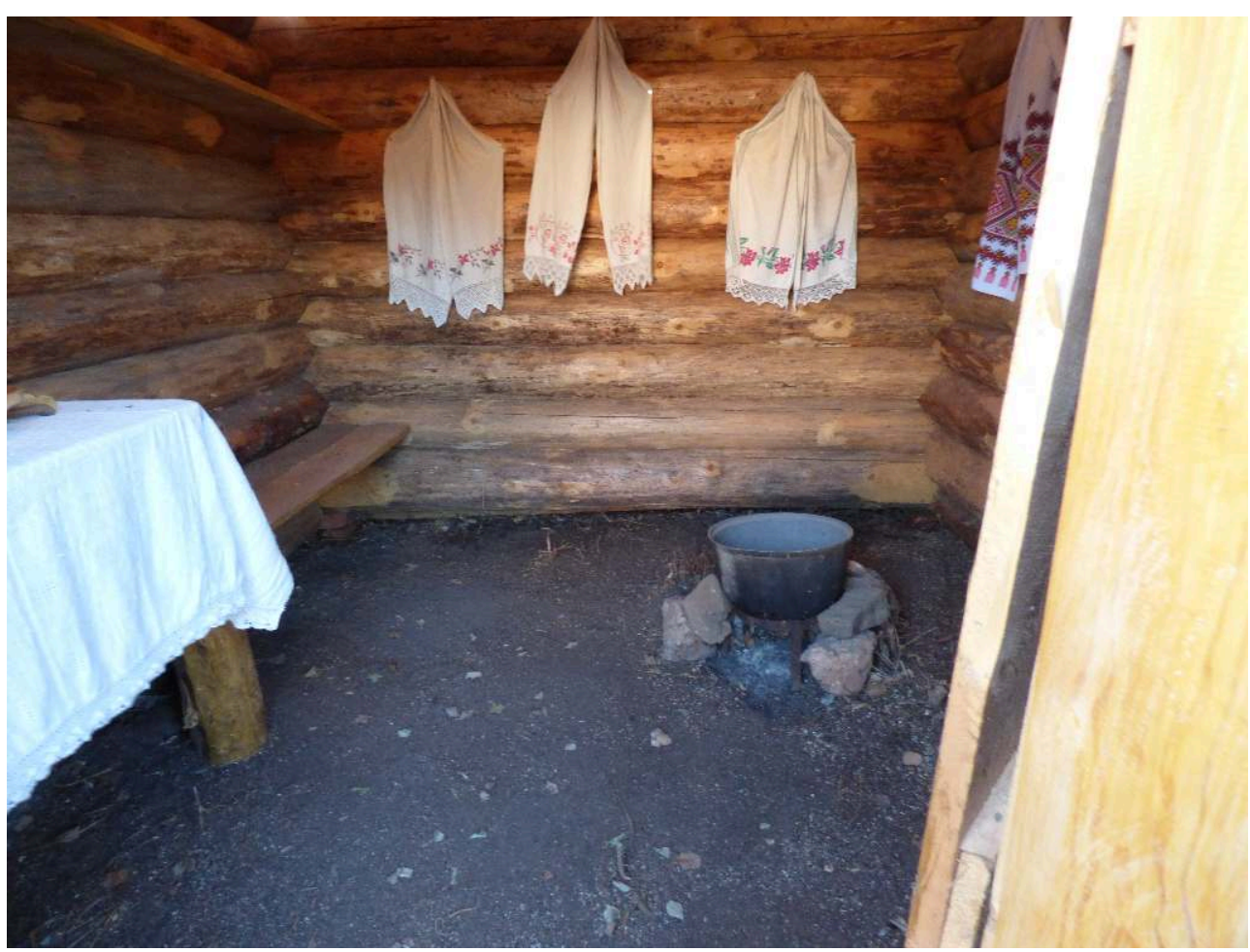

Intérieur de la kuala.

Photo Eva Toulouze, 4/2015.

Toujours est-il que ce village a une pratique des rituels traditionnels qui ne s'est pas interrompue pendant la période soviétique. Le village a plusieurs lieux sacrés, où ont lieu à différents moments de l'année des cérémonies. Ceci explique entre autre que les jeunes, comme Sasha, soient bien au courant des rituels: ils les ont vus pratiquer depuis leur enfance. Je m'interroge pourquoi, alors que je fréquente ce village depuis vingt ans, je n'en ai jamais entendu parler. Sans doute parce que je n'ai pas posé de questions à ce sujet, celui-ci fait partie de mes thèmes de recherche seulement depuis quelques années. Mais sans doute aussi parce que la période n'était pas propice. Je crois que la population suivait ses traditions sans en faire tout un plat, sans doute pas entièrement en secret, mais sans s'afficher non plus. Aujourd'hui, les choses ont changé. En effet, le village a commencé à s'afficher. Le centre de la culture oudmourte a changé les choses : il a proclamé la spécificité du village et ouvre aux touristes sa vie spirituelle.

30 C'est un choix. Tout le monde n'est pas d'accord avec ce choix : beaucoup considèrent que c'est une mascarade et qu'il vaut mieux ne pas faire un rituel que de l'inventer, si on n'est pas sûr de la manière dont les choses étaient faites. On les comprend. S'ils n'ont pas été consultés et si l'ouverture aux touristes les rend envahissants, les gens peuvent avoir l'impression d'être floués de quelque chose qui était intime et réel pour se transformer en spectacle pour curieux, dont on ne peut pas être sûr qu'ils porteront un regard respectueux.

31 Ce n'est pas ma place de donner des leçons à qui que ce soit, je me permets simplement de donner mon avis en tant que chercheur. Il me semble que cette ouverture n'est pas très éloignée de l'esprit des «religions autochtones », un esprit non dogmatique, un 
esprit holistique et non conflictuel. C'est dommage si les habitants de Karamas Pel'ga, qui jusqu'ici avaient pratiqué ces rituels à leur manière et naturellement, se mettent à jouer pour le regard de l'autre. Je ne sais pas si c'est le cas, je n'ai pas assisté à ces cérémonies. Ce serait dommage, et ce serait inutile. Car la nature du spirituel dans les cérémonies animistes n'est pas de la même nature que le spirituel chrétien.

Les habitants d'Uddjadi sont tous baptisés. L'évangélisation forcée est passée par là. Mais c'était il y a longtemps. Maintenant, la présence de l'icône dans l'angle de la grande pièce est parfaitement naturelle, qu'on soit activement croyant ou non (je l'ai trouvée dans toutes les maisons où je suis entrée). Et la Pâque sera fêtée de manières différentes, mais non contradictoires à Uddjadi. J'ai enregistré longuement Vassa, mais je me promets de venir dans ce village assister ou plutôt participer à ces festivités. Cela me permettra de me faire une opinion autre que par ouï-dire.

\section{Kuzebaevo}

Là aussi, je n'ai pas pu me permettre un séjour long à Kuzebaevo. Mais il fallait que j'y aille, ne serait-ce que pour, comme pour Uddjadi, une après-midi, une soirée et une matinée. En effet, mes amis de Kuzebaevo avaient appris ma venue et ont beaucoup insisté pour que je vienne. En effet, la dernière fois que je les avais vus, comme je l'ai relaté en 2011, les choses ne s'étaient pas très bien passées. Je n'avais pas été bien accueillie à la cérémonie de Bydžym kuala le 12 juillet et si l'atmosphère s'était nettement améliorée par la suite, cette réticence a sans doute été le déclencheur de mon intérêt pour l'étude de la religion oudmourte et m'a conduite à chercher un nouveau terrain chez les Oudmourtes du Bachkortostan. Si eux insistaient pour que je vienne, je n'avais pas le droit de ne pas leur répondre.

Cette fois-ci il n'y avait aucune cérémonie particulière, j'étais simplement accueillie chez eux comme une amie. Nous étions trois, mon ami Sanko et un chauffeur, Ruslan, un jeune garçon oudmourte qui avait accepté de nous conduire et de passer une journée ainsi à la campagne.

Notre premier thème de conversation a été un thème douloureux pour mes hôtes : les transformations que subit l'école. En effet tous deux sont enseignants. Lena est institutrice, Slava enseignant de travaux manuels. Tous deux sont malheureux dans leur boulot. Il est vrai qu'ils ne sont pas loin de l'âge de la retraite. Les changements les agressent de plein fouet : la «bureaucratie » scolaire s'est développée puissamment ces dernières années, de sorte que les enseignants passent un temps considérable à remplir des papiers. Tout le monde s'en plaint, pas seulement à Kuzebaevo. Mais manifestement dans ce village la situation a atteint le seuil du conflit. Mes amis refusent de remplir ces papiers qui leur empoisonnent la vie. En même temps, les classes sont de plus en plus difficiles. Vue par eux la situation est d'une indifférence totale des élèves, qui «ont tout » et qui ne s'intéressent à rien. Donc un travail plus difficile, sans doute avec un public qu'ils ont du mal à comprendre, et compliqué par une bureaucratie incompréhensible, et qui les oblige à utiliser un ordinateur qu'ils ne maîtrisent pas... Alors que les enfants sont grands et ont quitté la maison (les parents ne construisent pas moins une maison pour chacun d'entre eux à différents endroits), que les journées pourraient être moins lourdes, ce conflit les rejette en marge du village et de leur groupe de socialisation, des enseignants plus jeunes, qui bon gré mal gré accompagnent le mouvement et ne prennent pas le contrepied aussi résolument. 

seulement) rituels pour les Oudmourtes. Nous nous sommes installés dans la cuisine. Soudain Slava s'est levé, il est monté dans les chambres, en a descendu une robe blanche à broderies rouges, et l'a revêtue. Slava, il y a quatre ans, était vös'as' (officiant) pour l'un des clans du village, Lud, et il avait invité mes amis hommes à passer la nuit avec lui à une cérémonie réservée aux hommes. Il a donc revêtu son vêtement de vös'as', a noué une longue ceinture et en a donné une à Sanko pour qu'il l'accompagne. Il a posé sur une serviette une assiette avec trois taban' et est sorti, suivi par Sanko. Ruslan avait été invité, mais avait décliné l'invitation. J’y serais bien allée, mais j'étais coincée derrière la table. Me dégager aurait signifié bouleverser l'ordre des choses, et ce pour sortir et aller photographier... J'ai estimé que ce n'était pas ce que je voulais faire. J'ai donc fait confiance au regard de Sanko et je suis restée à ma place. Je pense que j'ai eu raison. Je ne suis pas tombée dans le piège de l'exotisme et je leur ai montré que je prenais l'acte de Slava avant tout pour ce qu'il est : un signe de bienvenue, et pas pour une mascarade. Sanko m'expliquera plus tard qu'ils sont sortis dans la cour et que Slava s'est tourné dans la direction du lieu sacré de son clan. Il a dit les paroles rituelles "Oste Inmare », qui sont une invocation au dieu Inmar. Sanko s'est tenu derrière lui. Toute prière a été dite mentalement. Et puis ils sont revenus dans la cuisine, Slava a jeté dans le four où les braises étaient incandescentes trois morceaux de taban' (photo 9) et il nous en a donnés à nous, tous les autres, Sanko comme les deux cuisinières, Lena et une amie, Ruslan et moi.

Photo 9

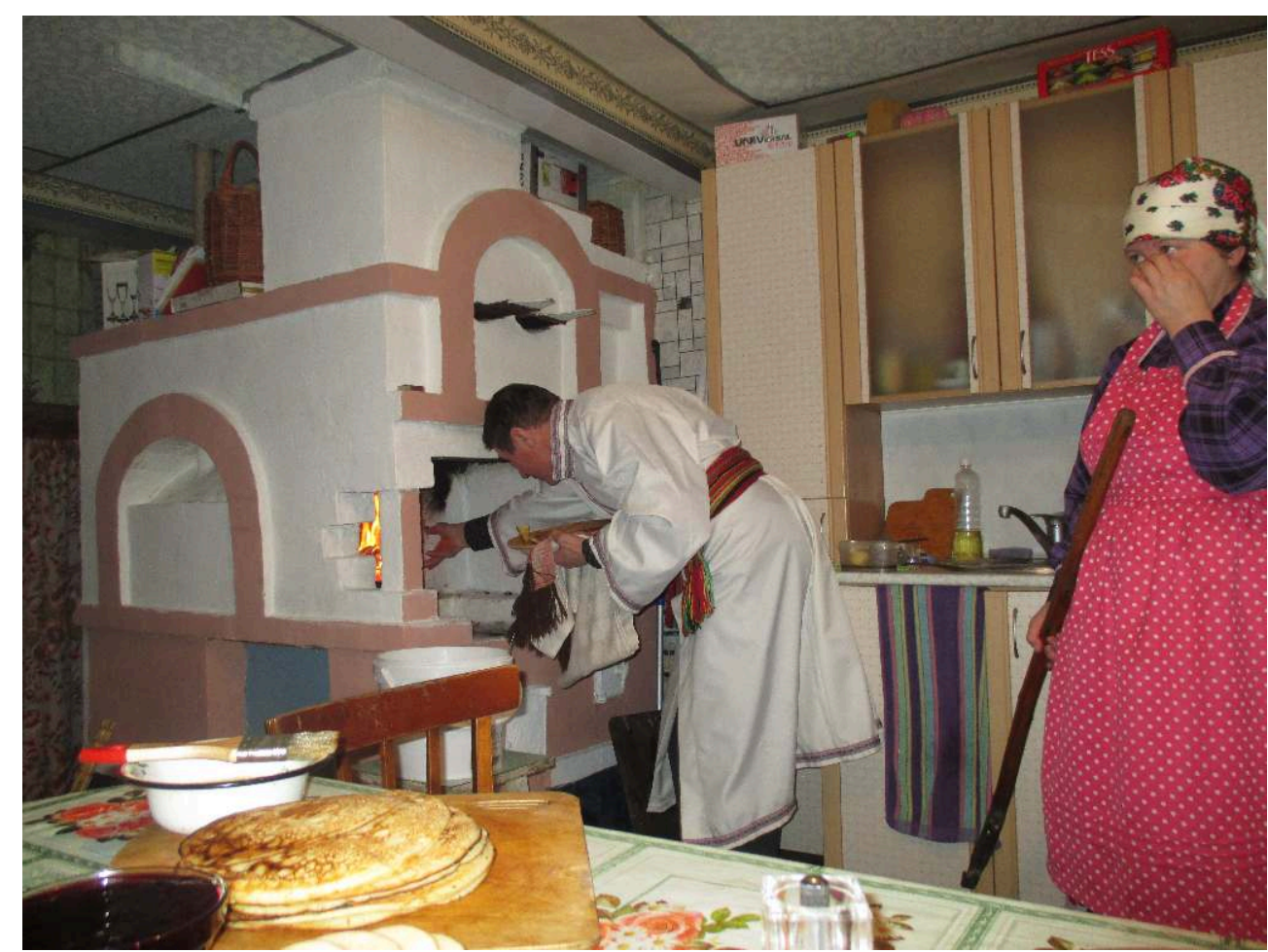

Kuzebaevo. Le vös'as jette dans le feu des morceaux de crêpes, après avoir dit une prière. Photo Eva Toulouze, 4/2015.

Et puis nous avons parlé. Slava a répondu aux questions que j'ai enregistrées sur ma caméra. Le village de Kuzebaevo, à l'inverse de Karamass-Pelga, est un village surtout 
d'Oudmourtes non baptisés. Cette image n'est pas rigoureuse aujourd'hui. Une partie de la population s'est fait baptiser. Lena m'a avoué qu'elle s'était fait baptiser adolescente parce que sa marraine avait une très belle écharpe qu'elle espérait avoir, et qui a perdu tout intérêt pour elle au bout de quelques mois... Pourquoi m'a-t-elle raconté cette histoire, incontestablement vraisemblable? Était-ce pour me dire que cet acte n'avait pas réellement été motivé par des considérations religieuses? Pour dévaloriser son identité d'orthodoxe? Une autre raison, que je n'ai pas explorée, fait qu'aujourd'hui il n'y a pas que des Oudmourtes non baptisés à Kuzebaevo : c'est que les protestants y ont été actifs. C'est Sanko qui m'en a informée. Ce sera un point à ne pas omettre dans mes prochaines rencontres.

Toujours est-il que la position défensive que j'avais perçue il y a quatre ans n'a pas disparu. J'ai essayé, dans mes entretiens, de mieux la comprendre. Est-ce que l'Église orthodoxe s'est montrée ces derniers temps particulièrement agressive contre lesdits «païens »? Il faudrait aller plus loin bien sûr, et il serait important pour moi de rencontrer des prêtres orthodoxes, à commencer par Mihail Atamanov, mais aussi d'autres dans les localités. J'ai d'ailleurs appris qu'autour du village d'Izgurt il y a des prêtres orthodoxes "dissidents" et, d'après Sanko, "bienveillants » envers les Oudmourtes pratiquant l'orthodoxie telle qu'ils la conçoivent...

On m'a parlé d'un article paru dans la presse russe: "un écrivain moscovite bon marché » (je cite) est allé les voir, ils l'ont reçu, ont répondu à toutes ses questions et il a fini par écrire un texte qui les présente sous un mauvais jour, qui se moque d'eux. Il aurait écrit qu'Inmar mange du canard pour son déjeuner... J'ai recherché ce texte. J'en ai trouvé un effectivement, où le canard était associé au déjeuner d'Inmar. Je ne suis pas seule à avoir trouvé ce texte inoffensif - mes autres amis oudmourtes ont été étonnés que ce texte ait été si mal reçu.

Je le reproduis ici :

Mais si vous voulez non pas un paganisme de musée, mais un paganisme véritable, il n'y a qu'une solution : aller à Kuzebaevo. Le raïon d'Alnaši, où se trouve ce village, est l'un des rares endroits dans la république où le paganisme n'a pas été revitalisé, il est resté vivant jusqu'à aujourd'hui. Ici, sous un grand saule, au bord de la rivière, se balancent au vent des petits sacs bien tendus au salpêtre, pleins d'os et de crânes. Les habitants d'ici, passant à l'oudmourte, nous racontent à l'envi l'histoire de leurs sanctuaires, où le grand sacerdote asperge le feu du sang des victimes, pour gagner la bienveillance du dieu Inmar. Mais les visiteurs n'ont pas de raisons d'avoir peur. La divinité préfère des canards et des moutons pour son déjeuner. Au village, il y a aujourd'hui trois clans, qui ont chacun ses traditions et ses rituels. Il y a encore une cinquantaine d'années, beaucoup priaient dans leurs kuala, leurs sanctuaires domestiques. S'il avait besoin de converser avec Dieu, le père de famille revêtait sa ceinture sacrale et se rendait dans la maisonnette sans plancher ni plafond pour faire un gruau rituel avec de la viande sacrificielle. À côté du foyer étaient suspendues les icônes et se trouvait un appareil de bouilleur de cru, d'où sortait une forte kumyška. Celle-ci était vue comme un don divin, on n'avait pas le droit de l'utiliser en dehors des rituels. Hélas, depuis beaucoup a changé. Les dizaines de divinités du panthéon oudmourte se sont fondues dans le bon Inmar et le méchant Šajtan, les traditions païennes avec les traditions chrétiennes et la kumyška est devenue partie intégrante de toutes les tables festives. Les sanctuaires domestiques se sont vidés depuis longtemps. Les esprits qui les habitaient ont redescendu le cours des fleuves et ne troublent plus les rêves des maîtres de la kuala. Maintenant, tout le clan se rassemble pour des cérémonies trois fois par an: au printemps à Pâques, à la saint Pierre le 12 juillet, et à l'Intercession le 14 octobre. Les cérémonies ont lieu dans un enclos, le lud, ou bien dans la grande kuala collective. Les 
sacerdotes abattent les canards sacrificiels, le gruau sacré bout dans les chaudrons, et les paysans, s'inclinant à terre prient Inmar. Presque comme jadis. Il est vrai que récemment, à Kuzebaevo, le parquet a débarqué et a exigé des papiers pour la kuala. Ils avaient découvert que quelqu'un avait construit dans la forêt. Ni arpentage, ni numéro de cadastre, Les villageois ont dû emmener sur un tracteur les représentants du Bureau des inventaires. Après quoi, ils ont eu la paix.

http://project2.strana.ru/route7/

Je m'interroge : y a-t-il dans ce texte quelque chose de méprisant vis-à-vis des habitants de Kuzebaevo ou de leurs croyances? Certes, le passage sur le déjeuner d'Inmar fait appel à un humour d'un goût douteux. Mais autrement, le texte semble plutôt informatif. Il n'est pas outre mesure empathique, car il regarde de l'extérieur, mais il semble quand même plutôt sympathiser avec les gens de Kuzebaevo. Or ce texte a suscité les foudres de mes hôtes. Et du coup, j'ai peur : que penserait Lena du texte que je suis en train d'écrire ici? Qu'y trouverait-elle de blessant? Or, vraiment, je n'éprouve à l'égard de mes hôtes de Kuzebaevo que du respect. J'ai beau être issue d'une autre tradition, je m'incline face à celle des Oudmourtes, qui porte en avant des valeurs de tolérance que j'aimerais trouver dans le monde qui m'entoure. Mais je ne regarde pas les choses uniquement de leur point de vue. J'essaye. Et je suis troublée.

41 Je pense qu'il faut que je passe plus de temps à Kuzebaevo, plus de temps avec mes amis, pour comprendre ce qui les trouble. Mais je ne peux pas exclure que l'habitude d'être seuls contre tous, d'être montrés du doigt et de subir la ségrégation les amène à voir des ennemis là où il n'y en a pas réellement.

Donc, il y a du travail pour mes prochains séjours, si je veux mieux comprendre la vision du monde des Oudmourtes d'aujourd'hui.

\section{NOTES}

1. Voir. par exemple

http://www.svoboda.org/content/article/26935642.html

2. À noter que Wikipédia évoque aujourd'hui cette affaire en disant que les koulaks ont organisé des punitions corporelles contre les paysans pauvres, reprenant, 25 ans après l'écroulement de l'Union soviétique, le vocabulaire et les mythes de lutte des classes de l'époque de la collectivisation

https://ru.wikipedia.org/wiki/ \%D0\%9B\%D1\%83\%D0\%B4\%D0\%BE\%D1\%80\%D0\%B2\%D0\%B0\%D0\%B9 


\section{INDEX}

Index géographique : Bachkortostan (République), Fédération de Russie, Izgurt, Iževsk, Karamas-Pel'ga, Kijasovo, Kuzebaevo, Ludorvaj, Moscou, Oudmourtie (République), SaintPétersbourg, Tatarstan (République)

Thèmes : anthropologie

Keywords : Travel, Transport, Rural Life, Scientific Research, Religious Life Index chronologique : XXIe siècle

nomsmotscles Itelmènes, Oudmourtes, Bessermans

motscleset reisimine, transport, maaelu, teadus, usuelu

motsclesru ПУТЕШЕСТВИЕ, ТРАНСПОРТ, ДЕРЕВЕНСКАЯ ЖИЗНЬ, РЕЛИГИОЗНАЯ ЖИЗНЬ

Mots-clés : voyages, transports, vie rurale, recherche scientifique, vie religieuse 